\title{
Influence of Purge Gas Flow and Heating Rates on Volatile Organic Compound Decomposition during Regeneration of an Activated Carbon Fiber Cloth
}

\author{
Saeid Niknaddaf ${ }^{1}$, John D. Atkinson ${ }^{1,2}$, Abedeh Gholidoust ${ }^{1}$, Mohammadreza Fayaz ${ }^{1}$, \\ Rania Awad ${ }^{1}$, Zaher Hashisho1*, John H. Phillips ${ }^{3}$, James E. Anderson ${ }^{4}$, Mark Nichols ${ }^{4}$ \\ ${ }^{1}$ University of Alberta, Department of Civil and Environmental Engineering, Edmonton, AB T6G 1H9, \\ Canada \\ ${ }^{2}$ State University of New York - University at Buffalo, Department of Civil, Structural, and \\ Environmental Engineering, Buffalo, NY 14260 USA \\ ${ }^{3}$ Ford Motor Company, Environmental Quality Office, Dearborn, MI 48126 USA \\ ${ }^{4}$ Ford Motor Company, Research and Advanced Engineering, Dearborn, MI 48121 USA \\ * Corresponding author: Tel.: +1-780-492-0247; Fax: +1-780-492-0249. E-mail: hashisho@ualberta.ca
}




\section{ACFC COMPOSITION}

\subsection{CHNS Elemental Analysis}

A CHNS elemental analyser (Vario MICRO) was used to characterize the bulk composition (carbon, hydrogen, nitrogen, sulfur, and oxygen) of regenerated ACFC samples with highest and lowest heel buildup. Oxygen in the sample is determined by mass difference $(100 \%-\mathrm{C}$ wt $\%-$ $\mathrm{H}$ wt $\%-\mathrm{N}$ wt $\%-\mathrm{S}$ wt $\%$ ), assuming that ash content is negligible. The detection limits for the analyzer were $0.25 \%$ for carbon, nitrogen, hydrogen and oxygen, and $0.27 \%$ for sulfur.

Table S1. Bulk composition of heat treated and regenerated ACFC after 5 TMB adsorption/regeneration cycles with highest (regenerated at 0.1 SLPM $\mathrm{N}_{2}$ ) and lowest (regenerated at 5 SLPM $N_{2}$ )

\begin{tabular}{lccccc}
\hline & $\begin{array}{c}\text { Heel } \\
(\%)\end{array}$ & $\begin{array}{c}\text { Nitrogen } \\
(\%)\end{array}$ & $\begin{array}{c}\text { Carbon } \\
(\%)\end{array}$ & $\begin{array}{c}\text { Hydrogen } \\
(\%)\end{array}$ & $\begin{array}{c}\text { Sulfur } \\
(\%)\end{array}$ \\
\hline Heat treated ACFC & 0.0 & 0.1 & 94.7 & 0.5 & 0.0 \\
\hline 5 SLPM & 1.4 & 0.1 & 95.4 & 0.6 & 0.0 \\
\hline 0.1 SLPM & 14.6 & 0.2 & 93.4 & 0.7 & 0.0 \\
\hline
\end{tabular}




\subsection{X-ray Photoelectron Spectroscopy}

X-ray photoelectron spectroscopy (XPS, Kratos AXIS 165) was used to characterize surface composition of regenerated ACFC samples. The instrument operated with mono Al Ka radiation at $210 \mathrm{~W}$ and $14 \mathrm{kV}$, under ultrahigh vacuum $\left(10^{-9}\right.$ Torr) with detection limit is $0.1 \%$. Spectra were calibrated to a $\mathrm{C}$ 1s peak location of $284.5 \mathrm{eV}$.

Table S2. Surface composition of ACFC Samples after 5 TMB adsorption/regeneration cycles with highest (regenerated at 0.1 SLPM $N_{2}$ ) and lowest (regenerated at 5 SLPM $N_{2}$ )

heel buildup

\begin{tabular}{|c|c|c|c|c|c|c|c|c|}
\hline & $\begin{array}{c}\text { Heel } \\
(\%)\end{array}$ & $\begin{array}{c}\text { Nitrogen } \\
(\%)\end{array}$ & $\begin{array}{c}\text { Carbon } \\
(\%)\end{array}$ & $\begin{array}{r}\text { Carbor } \\
\text { composit }\end{array}$ & $\begin{array}{l}\text { d } \\
\%)\end{array}$ & $\begin{array}{c}\text { Oxygen } \\
(\%)\end{array}$ & $\begin{array}{r}\text { Oxyge } \\
\text { compos }\end{array}$ & \\
\hline \multirow{4}{*}{5 SLPM } & \multirow{4}{*}{1.4} & \multirow{4}{*}{2.74} & \multirow{4}{*}{92.4} & $\mathrm{C}-\mathrm{C}$ & 55 & \multirow{4}{*}{4.85} & O1S & 52 \\
\hline & & & & $\mathrm{C}-\mathrm{O}$ & 27 & & $\mathrm{O}-\mathrm{C}$ & 39 \\
\hline & & & & $\mathrm{C}=\mathrm{C}$ & 1.5 & & $\mathrm{O}=\mathrm{C}$ & 8 \\
\hline & & & & $\mathrm{O}-\mathrm{C}=\mathrm{O}$ & 16 & & & \\
\hline \multirow{4}{*}{ 0.1 SLPM } & \multirow{4}{*}{14.6} & \multirow{4}{*}{2.59} & \multirow{4}{*}{92.4} & $\mathrm{C}-\mathrm{C}$ & 60 & \multirow{4}{*}{4.78} & O1S & 62 \\
\hline & & & & $\mathrm{C}-\mathrm{O}$ & 28 & & $\mathrm{O}-\mathrm{C}$ & 12 \\
\hline & & & & $\mathrm{C}=\mathrm{C}$ & 0.6 & & $\mathrm{O}=\mathrm{C}$ & 24 \\
\hline & & & & $\mathrm{O}-\mathrm{C}=\mathrm{O}$ & 11 & & & \\
\hline
\end{tabular}

